\title{
Genetic Landscape of congenital myasthenic syndromes from Turkey: Novel mutations and clinical insights
}

\author{
Uluç Yiş ${ }^{1}$, Kerstin Becker ${ }^{2}$, Semra Hız Kurul ${ }^{1}$, Gökhan Uyanik ${ }^{3,4}$, Erhan Bayram ${ }^{1}$, Göknur \\ Haliloğlu $^{5}$, Ayşe İpek Polat ${ }^{1}$, Müge Ayanoğlu ${ }^{1}$, Derya Okur ${ }^{1}$, Ayşe Fahriye Tosun ${ }^{6}$, Gül \\ Serdaroğlu ${ }^{7}$, Sanem Yilmaz ${ }^{7}$, Haluk Topaloğlü ${ }^{5}$, Banu Anlar ${ }^{5}$, Sebahattin Cirak $^{2}$, and \\ Andrew G. Engel ${ }^{8}$ \\ ${ }^{1}$ Dokuz Eylül University, School of Medicine, Department of Pediatrics, Division of Child \\ Neurology, İzmir, TURKEY \\ ${ }^{2}$ Cologne University, Department of Pediatrics, Center for Molecular Medicine Cologne, Cologne, \\ GERMANY \\ ${ }^{3}$ Center for Medical Genetics, Hanusch Hospital, Vienna, AUSTRIA \\ ${ }^{4}$ Medical Faculty, Sigmund Freud University, Vienna, AUSTRIA \\ ${ }^{5}$ Hacettepe University, School of Medicine, Department of Pediatrics, Division of Child Neurology, \\ Ankara, TURKEY \\ ${ }^{6}$ Adnan Menderes University, School of Medicine, Department of Pediatrics, Division of Child \\ Neurology, Aydın, TURKEY \\ ${ }^{7}$ Ege University, School of Medicine, Department of Pediatrics, Division of Child Neurology, İzmir, \\ TURKEY \\ ${ }^{8}$ Mayo Clinic, Rochester, Department of Neurology, MN 55905, USA
}

\begin{abstract}
Congenital myasthenic syndromes (CMS) are clinically and genetically heterogeneous disorders of neuromuscular transmission. Most are treatable, but certain subtypes worsen with cholinesterase inhibitors. This underlines the importance of genetic diagnosis. Here, we report on cases with genetically proven CMS from Turkey. We retrospectively reviewed our experience of all patients with CMS, referred over a 5-year period (2011-2016) to the Child Neurology Department of Dokuz Eylül University, Izmir, Turkey. In addition, PubMed was searched for published cases of genetically proven CMS originating from Turkey. In total, we identified 43 (8 new patients, 35 recently published patients) cases. Defects in the acetylcholine receptor ( $n=15$;
\end{abstract}

Corresponding author. Uluç Yiş, MD, Dokuz Eylül University, School of Medicine, Department of Pediatrics, Division of Child Neurology, İzmir, TURKEY, ulyis@yahoo.com, Tel: 0090232 4126216, Fax: 00902324126005.

Author Contributions

UY, the corresponding author, wrote drafts for the manuscript. Each draft and the final manuscript were reviewed and approved by each listed author and accepted for submission for publication.

Declaration of Conflicting Interests

The authors declared no potential conflicts of interest with respect to the research, authorship, and or publication of this article.

Ethical Approval

This study was approved by Dokuz Eylül University Institutional Review Board (2016/23-16). 
$35 \%)$ were the most common type, followed by synaptic basal-lamina associated $(\mathrm{n}=14 ; 33 \%)$ and presynaptic syndromes $(\mathrm{n}=10 ; 23 \%)$. We only had 3 cases $(7 \%)$ who had defects in endplate development. One patient had mutation GFPT1 gene $(\mathrm{n}=1 ; 2 \%)$. Knowledge on CMS and related genes in Turkey will lead to prompt diagnosis and treatment of these rare neuromuscular disorders.

\section{Keywords}

congenital myasthenic syndromes; genetic diagnosis; Turk

\section{Introduction}

Congenital myasthenic syndromes (CMS) are a group of rare disorders resulting from defects in the structure and function of the neuromuscular junction. Patients with CMS usually have fatigable muscle weakness and additional symptoms depending on the type of protein affected. The diagnosis depends on clinical findings and detection of abnormal jitter on single fiber electromyography and/or decrement in repetitive nerve stimulation. CMS are genetically heterogeneous, mostly inherited in autosomal recessive fashion. To date, more than 20 causative genes have been reported. ${ }^{1}$ These genes mainly affect proteins of the postsynaptic neuromuscular junction, mainly the subunits of acetylcholine receptor. Despite advances in genetic medicine, $20 \%$ of cases with CMS lack a genetic diagnosis. ${ }^{1}$ Knowledge on the phenotype, related genes and course of the syndrome would assist the physicians in correct diagnosis and management of the patient. The aim of this study is to document the clinical findings and related mutations in genetically proven cases of CMS reported from Turkey. Patients of Turkish origin living in and reported from other countries were also included in our review of the literature.

\section{Patients and Methods}

We retrospectively reviewed our experience of all patients with CMS, referred over a 5-year period (2011-2016) to the Child Neurology Department of Dokuz Eylül University, Izmir, Turkey. The diagnosis of CMS was based on clinical symptomatology (described below), absence of autoantibodies, electromyographic evidence of neuromuscular transmission defect and molecular genetic confirmation. Clinical data including age at onset and presenting symptoms, family history and/or recurrent infantile deaths, feeding difficulties and need for gastrostomy, need for non-invasive ventilation and/or tracheostomy, results of repetitive stimulation, muscle biopsy, genetic examinations and pharmacological management were retrieved from medical records. In addition, PubMed was searched for published cases of genetically proven CMS originating from Turkey.

\section{Targeted Gene Sequencing and Whole exome sequencing}

Most of the patients underwent targeted gene sequencing according to their clinical symptoms. Three patients underwent whole exome sequencing. Genomic DNA of the index patients was enriched for coding exons with Sure Select Human All Exon V6 library preparation Kit and sequenced on an Illumina High Seq 2000. Variants were called using the Cologne Center for Genomics in house analysis pipeline Varbank (https://varbank.ccg.uni- 
koeln.de/) and filtered according to the expected inheritance model for each patient. Variants and their cosegregation were validated by standard Sanger sequencing.

\section{Results}

\section{Patients from Dokuz Eylül University, Faculty of Medicine}

Eight cases were included in the study. Four cases had mutations in the acetylcholine receptor subunits ( 3 in epsilon (CHRNE), 1 in delta subunit $(C H R N D)$ ), one had mutation in the choline acetyltransferase (CHAT) gene ${ }^{2}$ and two had mutation in the collagenic tail of endplate acetylcholinesterase (COLQ) gene. One patient had mutation in the GFPT1 gene.

\section{Other patients of Turkish origin (living in Turkey or elsewhere)}

According to our literature search, 35 cases of CMS of Turkish origin have been reported. Eleven of these mutations are in the $\mathrm{AChR}$ epsilon subunit, twelve in $C O L Q$, seven in CHAT, two in MUSK, two in MYO9A, and one in $A G R N .^{3-12}$

\section{Patients with CHRNE and CHRND mutations}

A total of 14 patients had mutations in the CHRNE gene and one case had mutation in the CHRND gene. Eleven of these cases were previously reported. ${ }^{3}$ They all had ptosis, ophthalmoplegia and bulbar or limb involvement before 4 years of age, mostly at birth. Symptoms started at the age of 7 years in one patient. None of the patients showed progression of disease nor had respiratory crisis. Constant findings at advanced ages were ptosis and ophthalmoplegia. Four patients did not respond to anticholinesterase drugs and in the remaining patients, the response to anticholinesterase drugs were partial. Repetitive stimulation of the hypothenar muscle did not show decrement in 9 of 14 tested cases while single fiber electromyography showed increased jitter in all tested cases.

One patient harbored a homozygous mutation (p.L331F) in the AChR delta subunit. The parents were consanguineous. Symptoms started at birth with ptosis, ophthalmoplegia, bulbar weakness and apnea attacks. Respiratory findings and bulbar weakness decreased with advancing age. She is now 7 years old and the primary findings are ptosis and ophthalmoplegia. Repetitive stimulation showed a greater than $10 \%$ decrement in abductor digiti minimi muscle. She benefited from pyridostigmine bromide. 3,4 diaminopyridine (3,4 DAP) and salbutamol were not tried. Her aunt had hypotonia and ptosis and died at the age of one year because of respiratory insufficiency.

\section{Patients with COLQ mutations}

A total of 14 cases had mutations in $C O L Q$ gene. Twelve patients were previously reported. ${ }^{4-8}$ The median age was 8.9 years (18 months -17 years). The disease manifested from birth to up to two years of age. Three patients were hypotonic at birth. Seven patients had motor developmental delay. Ptosis, restricted eye movements, facial weakness, bulbar weakness and respiratory crisis were the main presenting findings. Proximal muscle weakness $(n=12)$, axial muscle weakness $(n=7)$, scoliosis $(n=5)$ and neck weakness $(n=5)$ were other important findings. Six patients had respiratory crises and two patients had tracheostomy. Six patients had decremental EMG at repetitive stimulation and a repetitive 
compound muscle action was observed in one patient. Two patients had slow pupillary response. Most patients had worsening of their symptoms with acetylcholinesterase (AChE) inhibitory therapy. Salbutamol, ephedrine and 3,4-DAP were the most effective treatments. One patient did not benefit from salbutamol and ephedrine treatment. Most patients were motor handicapped and needed assisted ventilation during the follow up. Seven patients were homozygous for the p.W148* mutation, two were homozygous for the c.1082delC mutation, one patient was compound heterozygous for the mutations p.W148* and p.R236*, and one was homozygous for p.R236*. Other patients were homozygous for the mutations c. $1281 \mathrm{C}>\mathrm{T}$ (p.C427=; creation of a cryptic splice donor site), p.N309S and p.Arg227*, respectively.

\section{Patients with CHAT mutations}

Eight patients had mutations in $C H A T$ and all were previously reported. ${ }^{2,9}$ The median age at the time of diagnosis was 6.4 years (range: 6 months - 14 years). The disease manifested from the neonatal period to up to the age of two years. Ptosis, apnea, hypotonia, bulbar weakness, facial weakness, generalized weakness, exercise intolerance and crises with respiratory insufficiency were the presenting findings. In three patients, apneas were misdiagnosed as seizures leading to antiepileptic treatment. Seven patients were mentally retarded. Patients benefited from therapy with AChE inhibitory agents and 3,4-DAP. In one patient, apneic attacks responded to diazepam treatment. In half of the patients, no pathologic EMG decrements were recorded. Creatine kinase levels were normal in all. Muscle biopsy examinations in four patients were unremarkable or showed only nonspecific myopathic changes. The clinical course was normal in only one patient, and one patient died. The others had varying degrees of ptosis, psychomotor retardation, muscle weakness, bulbar weakness, and respiratory crises with intermittent ventilation. Two patients had tracheostomy. Seven patients were homozygous for the p.I336T mutation and one patient for the p.G417R mutation.

\section{Patients with AGRN mutations}

One patient had mutations in the $A G R N$ gene. ${ }^{10}$ This patient was a 3 -year-old boy who initially presented with isolated neck weakness and developed ptosis and restricted eye movements during the follow up period. His symptoms improved after pyridostigmine treatment. Ha had a homozygous point mutation (c.5023G >A; p.G1675S) in the $A G R N .^{10}$

\section{Patients with MUSK mutations}

Two siblings aged 13 and 16 years, previously reported, had homozygous $\mathrm{T}$ to A transition in exon 2 at nucleotide position 114 leading to an amino acid exchange (p.D38E) in the MUSK gene. ${ }^{11}$ The parents were not consanguineous. Main clinical findings were fatigable limb weakness from early childhood. Facial weakness was mild without bulbar and ocular symptoms. Repetitive stimulation of the accessory nerve resulted in more than $10 \%$ decrement. Creatine kinase levels were normal with minimal myopathic changes in muscle biopsy. A trial of oral pyridostigmine bromide worsened symptoms while oral salbutamol was effective. 


\section{Patients with MYO9A mutations}

Two previously reported siblings aged 5 and 11 years born to consanguineous parents had a homozygous missense mutation (p.D1698G) in the MYO9A gene. ${ }^{12}$ The family history included four previous children dying in the first year of life due to respiratory failure, feeding difficulties and hypotonia. The current patients had reduced fetal movements. Generalized hypotonia, ptosis, nystagmus, oculomotor apraxia, ophthalmoplegia, bulbar weakness and motor retardation were noticed from early age. One of the siblings also had mental retardation. Repetitive stimulation showed more than $10 \%$ decrement in the ala nasi muscle in the older sibling. Respiratory crises occurred after oral intake of 3,4 DAP and fluoxetine; however pyridostigmine bromide reduced the symptoms.

\section{Patients with GFPT1 mutations}

One patient had mutation in the GPFT1 gene (c.686-2A>G) in homozygous state. The patient was a 17-year-old boy who presented with limb girdle and axial weakness. He was the first child of consanguineous parents. His motor development was normal but the family noticed that he could not rise from the floor like his peers until the age of 13 months. At presentation, he was able to walk without support. Muscle power in upper and lower extremities were symmetrically $3 / 5$ and $4 / 5$ in proximal and distal muscles, respectively. $\mathrm{He}$ had prominent neck flexor weakness with mild scoliosis. Muscles were atrophic and deep tendon reflexes were absent. He also had pes cavus deformity. His mentality was normal. $\mathrm{He}$ had no ocular, facial or bulbar weakness. Serum creatine kinase was 1400 IU/L. Nerve conduction studies were normal but electromyography was myopathic. His muscle biopsy done at the age of 10 years showed dystrophic pattern. Genetic analysis for dystrophin, SEPN1 and LMNA were negative.

The mutations identified in patients from Turkey and their references are shown in Table 1.

\section{Discussion}

Congenital myasthenic syndromes are inherited disorders of neuromuscular transmission. They have been classified according to the location of the mutant protein as presynaptic, synaptic and postsynaptic. After 2011, whole-exome sequencing has led to the discovery of new CMS genes and to date more than 20 CMS disease genes have been identified. ${ }^{1}$ Classification based on a cohort of patients with CMS investigated at the Mayo Clinic between 1998 and 2004 showed that defects in AChR the most common cause of CMS followed by defects in endplate development and maintenance, synaptic basal laminaassociated CMS, and defect in presynaptic proteins. ${ }^{1}$ In another series of 680 patients with CMS, mutation rates were similar to those investigated at the Mayo Clinic. ${ }^{13}$ In our study of Turkish patients, mutations in $C H R N E$ which encoded the epsilon subunit of AChR were the most common followed by synaptic basal-lamina associated (COLQ mutation) and presynaptic syndromes (CHAT mutation). We only had 3 patients (two MUSK and one $A G R N$ ) who had defects in endplate development and maintenance which is the second most common cause of CMS in two large series. 
Most CMS stem from molecular defects in the muscle nicotinic acetylcholine receptor. The subunit composition at the adult endplate is $\mathrm{a}_{2} \beta \delta \varepsilon$. The genes coding the a (CHRNAI) and $\delta(C H R N D 1)$ are on chromosome 2 and those encoding the $\beta$ (CHRNB) and $\varepsilon$ (CHRNE) are located on chromosome $17 .{ }^{1}$ Mutations in CMS genes in a collective of 680 index patients showed that $49 \%$ of all mutations were caused by $C H R N E$ mutations. Only three patients $(<1 \%)$ had mutations in the CHRND gene. ${ }^{13}$ Most of the mutations found in the CHRNE gene are inherited in an autosomal recessive manner and result in a loss of function and deficiency of AChR at the end plate. The $1267 \mathrm{delG}$ mutation (annotation according to HGVS: c.1327delG) is a founder mutation of CHRNE gene and frequently found in South Eastern Europe, India/Pakistan and among Roma. ${ }^{13}$ In our study, the CHRNE mutations were located in different exons or introns and we noted no founder effect. Symptoms can begin in childhood, adolescence or adulthood. The clinical findings include hypotonia, delayed motor development, ptosis, ophthalmoplegia, exertion induced weakness, skeletal deformities, muscular atrophy, dysphagia and respiratory difficulty. This form of CMS is often misdiagnosed as autoimmune myasthenia gravis. There is a decremental EMG response after repetitive stimulation but not in all patients but single fiber electromyography shows increased jitter in all. ${ }^{14}$ Our patients' symptoms presented mostly started before 4 years of age. The main symptoms were ptosis and limited eye movements. The symptoms were not progressive and none of the patients had respiratory crises. Although the patients were improved by acetylcholinesterase inhibitors the response of our patients with mutations in $C H R N E$ was not entirely satisfactory. Patients with $C H R N D$ mutations have a more severe course with bulbar weakness and apneic attacks in the newborn period. Fortunately, apneic attacks and bulbar weakness decrease with increasing age and main symptoms in advanced age are ptosis and limited eye movements.

Mutations in $C O L Q$ were the second most common. In the series of Engel et al. 45 of 354 patients $\left(12.6 \%\right.$ ) had mutations in the COLQ. ${ }^{1}$ In the series of Abicht et al. 38 of 680 patients (6\%) had mutations in the COLQ gene. ${ }^{13}$ Most mutations in $C O L Q$ result in a total loss of protein function (frameshift or stop mutations) on both alleles. The c.1082delC mutation has been repeatedly identified in Turkey, Italy, Georgia and Czech Republic. ${ }^{13}$ However, in our study, p.W148* mutation was found in eight patients either in homozygous or compound heterozygous state pointing toward a founder mutation in Turkey. Two clinical phenotypes of with $C O L Q$ mutations have been described. ${ }^{15,16}$ The classical phenotype includes disease onset at birth, moderate to severe weakness and no response to esterase inhibitors. Patients typically have respiratory crises, involvement of ocular muscles, facial and generalized weakness. In the other phenotype, disease starts at 6-7 years of age and patients have mild weakness. The disease does not progress and cranial muscles are spared. There may also be other forms like a non-progressive disease with onset in infancy or a late onset form with rapid progression. ${ }^{6}$ Important clinical clues to ColQ deficiency are worsening with cholinesterase inhibitors, a repetitive compound action potential, and a slow pupillary light response. ${ }^{17}$ Beneficial effects of albuterol and ephedrine were observed in this type of CMS. The clinical findings of our patients were compatible with the classical phenotype of the disease. The disease course was also progressive with progressive scoliosis, need for assisted ventilation and wheelchair. The most effective treatments in our patient were albuterol and 3,4 DAP. 
The third most common mutation found in our study was $C H A T$ mutations. In the series of Engel et al. 5.1\% patients and in Abicht et al. $2 \%$ had mutations had mutations in the CHAT gene. ${ }^{1,13}$ Most molecular defects are missense mutations. ${ }^{13}$ Seven of 8 cases had p.I336T mutation, a founder mutation in Turkey. Symptoms start at birth or infancy in all cases and generally respond well to esterase inhibitors. The most differentiating symptom of this subtype of CMS is episodic apnea and worsening of myasthenic symptoms with infections, fever or stress. Apneic episodes are frequently misdiagnosed as intractable seizures and treated with antiepileptic drugs. In our study, only one case showed normal development while the remaining had psychomotor retardation. Central nervous system involvement may be due to recurrent hypoxic episodes. Routine $3 \mathrm{~Hz}$ repetitive stimulation is not helpful in the diagnosis of this subtype of CMS: none of our tested cases showed decrement to this test. High frequency stimulation at $10 \mathrm{~Hz}$ for 5 minutes followed by $3 \mathrm{~Hz}$ stimulation may demonstrate a pathological decrement. This procedure is not easy to perform in pediatrics and may need sedation. Patients generally respond well to 3,4 DAP and esterase inhibitors and these treatments should be initiated early after genetic confirmation to avoid secondary hypoxic brain damage. Interestingly, one of our cases responded to diazepam treatment during apneic episodes which were mistaken for seizures. ${ }^{2}$ We can speculate that diazepam reduces the mean firing frequency in the central nervous system that activates motor neurons in a way that the neuromuscular transmission becomes more efficient in muscles. The probability of successful neuromuscular transmission is increased when motor units are stimulated at a subnormal frequency.

One child had mutation in the $A G R N$ gene. Only a few patients with $A G R N$ mutation has been described. ${ }^{18,19}$ The disease may present with mild limb and facial muscle weakness and bilateral lid ptosis. In this type, diplopia, bulbar symptoms and dyspnea have never been reported. Long-term follow up showed no deterioration of muscle function and symptoms did not respond to cholinesterase inhibitors and 3,4-DAP. ${ }^{18}$ Another phenotype is more severe with weakness starting in early childhood, bilateral ptosis and respiratory insufficiency requiring tracheotomy. Response to cholinesterase inhibitors were moderate. ${ }^{19}$ The phenotype in our patient was interesting. This patient initially presented with a cervical myopathy and developed ptosis and ocular findings during the course of disease. ${ }^{10}$

The remaining four patients (two of each) had mutations in MUSK and MYO9A genes, respectively. MUSK mutations as a cause of CMS have been rarely reported. The clinical phenotype is limb girdle CMS unresponsive to esterase inhibitors. The eye movements are normal. Clinical findings also resemble DOK7 deficiency. ${ }^{10}$ MYO9A is the one of the newest genes described in the literature. It has been described in three patients, two of whom were siblings. The clinical findings start at birth with hypotonia, swallowing, chewing difficulties, and respiratory crises. Motor development is delayed and one of the patients was intellectually challenged. Interestingly, two siblings also had nystagmus and oculomotor apraxia. Cholinesterase inhibitors improved the patients' symptoms. ${ }^{11}$

A difficult to diagnose subgroup of CMS is characterized by limb girdle weakness with no ocular and facial involvement. Responsible genes in this group are DOK7 and GFPT1. GFTP1 is the key enzyme of the hexosamine pathway. Symptoms start at first decade with walking difficulties and weakness of shoulder or pelvic muscles. Patients do not have ocular, 
facial and bulbar weakness and respiratory problems. Fluctuation of weakness is daytimedependent and progression of the disease is slow. In contrast to classical myasthenia, serum creatine kinase is elevated in most cases and muscle biopsy specimens show tubular aggregates. Response to esterase inhibitors are positive in most of the cases. There is no obvious common mutation or mutation hotspot. It is not clear whether the origin of the neurotransmission defect is primarily synaptic or postsynaptic. ${ }^{20}$ Our patient presented with limb girdle and axial weakness with increased serum creatine kinase and a myopathic pattern on electromyography suggesting a muscular dystrophy. GFPT1 related myasthenia should also be considered in the differential diagnosis of unsolved limb girdle myasthenia cases. Axial weakness and atrophic pattern may be clinical clues for diagnosis.

Interestingly, no patients in our study had mutations in $R A P S N$ or $D O K 7$ which constitute $25 \%$ of CMS in other series. Both genes are important in endplate development and maintenance. On the other hand, they are autosomal recessively inherited. When we consider that the rate of consanguineous marriages is high in Turkey, it is hard to understand why no patients with mutations in these genes have been reported. We believe that number of patients with CMS in Turkey will increase with increasing knowledge of the disease and advances in genetic diagnosis.

A recent study from Israel showed that $R A P S N$ mutations were the most common cause of CMS followed by $C O L Q$ and $C H R N E$. The authors suggested that this information may be useful for physicians encountering suspected CMS in patients of Middle-Eastern origins in other parts of the world. Turkey is a country very close to Middle-Eastern and the structure of community is similar to Israel like different ethnicities and high level of consanguinity. However, CHRNE mutations were the most common cause of CMS in Turkey. Since next generation methods are increasingly available, this method will fasten the diagnosis of CMS cases where genetic mutations are heterogeneous. ${ }^{21}$

In conclusion, CMS are rare but important neuromuscular disorders. Most of them respond to medications like cholinesterase inhibitors, 3,4 DAP, ephedrine or salbutamol. On the other hand, these medications especially cholinesterase inhibitors and 3,4 DAP may worsen even lead to death in some subtypes of the disease. In Turkey, CMS patients with symptoms restricted to eye muscles can first be tested for AChR epsilon subunit; progressive axial weakness and worsening with pyridostigmine for $C O L Q$ and apnea in infancy for CHAT. Having knowledge of these rare disorders, their related genes and clinical findings in a specific geographic region should alert the physicians for prompt disease identification and treatment.

\section{Acknowledgments}

Funding

Genetic studies performed in Dr. Andrew Engel's laboratory were supported by NIH Grant NS6277. Dr. Sebahattin Cirak is funded by the German Research Foundation (DFG) (Emmy Noether Arbeitsgruppe). 


\section{References}

1. Engel AG, Shen XM, Selcen D, Sine SM. Congenital myasthenic syndromes; pathogenesis, diagnosis, treatment. Lancet Neurol. 2015; 14:420-434. [PubMed: 25792100]

2. Yis U, Kurul SH, Öztura İ, Özden Ö, Akıncı G, Dirik E. Temporary diazepam responsive apneic attacks and congenital myasthenic syndrome. J Child Neurol. 2009; 24:895-898. [PubMed: 19289695]

3. Ohno K, Anlar B, Özdirim E, Brengman JM, DeBleecker JL, Engel AG. Myasthenic syndromes in Turkish kinships due to mutations in acetylcholine receptor. Ann Neurol. 1998; 44:234-241. [PubMed: 9708546]

4. Güven A, Demirci M, Anlar B. Recurrent COLQ mutation in congenital myasthenic syndrome. Pediatr Neurol. 2012; 46:253-256. [PubMed: 22490774]

5. Duran GS, Uzunhan TA, Ekici B, Çıtak A, Aydınlı N, Çalışkan M. Severe scoliosis in a patient with COLQ mutation and congenital myasthenic syndrome: a clue for diagnosis. Acta Neurol Belg. 2013; 113:531-532. [PubMed: 23371844]

6. Mihaylova V, Müller JS, Vichez JJ, et al. Clinical and molecular genetic findings in COLQ-mutant congenital myasthenia gravis. Brain. 2008; 131:747-759. [PubMed: 18180250]

7. Wargon I, Richard P, Kuntzer T, et al. Long-term follow-up of patients with congenital myasthenic syndromes caused by COLQ mutations. Neuromuscul Disord. 2012; 22:318-324. [PubMed: 22088788]

8. Haliloğlu G, Demirci T, Alikaşifoğlu M, Aktaş D, Topaloğlu H. Congenital myasthenic syndromes due to COLQ mutations: Clues for diagnosis. Neuromuscul Disord. 2016; 26:S112.

9. Schara U, Christen HJ, Durmuş H, et al. Long-term follow-up in patients with congenital myasthenic mutations due to CHAT mutations. Eur J Ped Neurol. 2010; 14:326-333.

10. Karakaya M, Birsoy OC, Beggs AH, Topaloğlu H. A Novel missense mutation in the AGRN gene causing congenital myasthenic syndrome mimicking neck myopathy. J Clin Neuromuscul Dis. 2016 epub ahead of print.

11. Gallenmüller C, Müller-Felber W, Dusl M, et al. Salbutamol-responsive limb-girdle congenital myasthenic syndrome due to a novel missense mutation and heteroallelic deletion in MUSK. Neuromuscul Disord. 2014; 24:31-35. [PubMed: 24183479]

12. O'Connor E, Töpf A, Müller JS, et al. Identification of mutations in the MYO9A gene in patients with congenital myasthenic syndrome. Brain. 2016; 139(8):2143-2153. [PubMed: 27259756]

13. Abicht A, Dusl M, Gallenmüller C, et al. Congenital myasthenic syndromes: Achievements and limitations of phenotype-guided gene after gene sequencing in diagnostic practice: a study of 680 patients. Hum Mutat. 2012; 33:1474-1484. [PubMed: 22678886]

14. Lorenzoni PJ, Scola RH, Kay CS, Werneck LC. Congenital myasthenic syndrome: a brief review. Pediatr Neurol. 2012; 46:141-148. [PubMed: 22353287]

15. Ohno K, Brengman J, Tsujino A, Engel AG. Human endplate acetylcholinesterase deficiency caused by mutations in the collagen-like tail subunit (ColQ) of the asymmetric enzyme. Proc Natl Acad Sci USA. 1998; 95:9654-9659. [PubMed: 9689136]

16. Müller JS, Petrova S, Kiefer R, et al. Synaptic congenital myasthenic syndrome in three patients due to a novel missense mutation (T441A) of the COLQ gene. Neuropediatrics. 2004; 35:183-189. [PubMed: 15248101]

17. Engel AG, Ohno K, Sine M. Congenital myasthenic syndromes: progress over the past decade. Muscle Nerve. 2003; 27:4-25. [PubMed: 12508290]

18. Huze C, Bauche S, Richard P, et al. Identification of an Agrin mutation that causes congenital myasthenia and affects synapse function. AJHG. 2009; 85:155-167.

19. Maselli RA, Fernandez JM, Arredondo J, et al. LG2 Agrin mutation causing severe congenital myasthenic syndrome mimics functional characteristics of non-neural (z-) Agrin. Hum Genet. 2012; 131:1123-1135. [PubMed: 22205389]

20. Guergueltcheva V, Müller JS, Dusl M, et al. Congenital myasthenic syndrome with tubular aggregates caused by GFPT1 mutations. J Neurol. 2012; 259:838-850. [PubMed: 21975507] 
21. Aharoni S, Sadeh M, Shapira Y, et al. Congenital myasthenic syndromes in Israel: Genetic and clinical characterization. Neuromuscul Disord. 2016; 27:136-140. [PubMed: 28024842] 
Table 1

The mutations in congenital myasthenic syndrome genes identified in patients from Turkey

\begin{tabular}{|c|c|c|c|}
\hline Classification of CMS & Mutations & Published cases & References \\
\hline \multicolumn{4}{|l|}{ Presynaptic } \\
\hline \multirow[t]{3}{*}{ CHAT } & $\begin{array}{l}\text { c. } 1249 \mathrm{G}>\mathrm{A} \text { (1 case) } \\
\text { p.G417R }\end{array}$ & Yes & $\begin{array}{l}\text { Schara U, et al. Eur J Ped Neurol. 2010; } \\
\text { 14:326-333. }\end{array}$ \\
\hline & c. $1007 \mathrm{~T}>\mathrm{C}$ ( 7 cases $)$ & Yes & $\begin{array}{l}\text { Schara U, et al. Eur J Ped Neurol. 2010; } \\
\text { 14:326-333. }\end{array}$ \\
\hline & p.I336T & & $\begin{array}{l}\text { Yis U, et al. J Child Neurol. 2009; } \\
\text { 24:895-898. }\end{array}$ \\
\hline \multirow[t]{2}{*}{ MYO9A } & c. $5093 \mathrm{~A}>\mathrm{G}(2$ cases $)$ & & $\begin{array}{l}\text { O'Connor, et al. Brain 2016; 139:2143- } \\
2153 . \text { E }\end{array}$ \\
\hline & p.D1698G & & \\
\hline \multicolumn{4}{|l|}{$\begin{array}{l}\text { Synaptic basal lamina } \\
\text { associated }\end{array}$} \\
\hline \multirow[t]{15}{*}{ COLQ } & c. $444 \mathrm{G}>\mathrm{A}(7$ cases $)$ & Yes & $\begin{array}{l}\text { Güven A, et al. Pediatr Neurol. 2012; } \\
\text { 46:253-256. }\end{array}$ \\
\hline & p.W148* & & $\begin{array}{l}\text { Wargon I, et al. Neuromuscul Disord. } \\
2012 ; 22: 318-324 .\end{array}$ \\
\hline & & & Halilioğlu G, et al. 2016; 26:S112 \\
\hline & c.1082delC ( 2 cases) & Yes & $\begin{array}{l}\text { Mihaylova V, et al. Brain 2008; 131:747- } \\
759 \text {. }\end{array}$ \\
\hline & p.P361Lfs*64 & & $\begin{array}{l}\text { Duran GS, et al. Acta Neurol Belg. 2013; } \\
\text { 113:531-532. }\end{array}$ \\
\hline & c. $706 \mathrm{C}>\mathrm{T}$ (1 case $)$ & Yes & $\begin{array}{l}\text { Mihaylova V, et al Brain 2008; 131:747- } \\
759 \text {. }\end{array}$ \\
\hline & p.R236* & & \\
\hline & c. $1281 \mathrm{C}>\mathrm{T}(1$ case $)$ & Yes & $\begin{array}{l}\text { Wargon I, et al. Neuromuscul Disord. } \\
2012 ; 22: 318-324 .\end{array}$ \\
\hline & p.C427= (splice mutation) & & \\
\hline & c.679C $>\mathrm{T}(1$ case $)$ & Yes & $\begin{array}{l}\text { Wargon I, et al. Neuromuscul Disord. } \\
\text { 2012; 22:318-324. }\end{array}$ \\
\hline & p.R227* & & \\
\hline & c. $1169 \mathrm{~A}>\mathrm{G}(1$ case $)$ & No (this study) & \\
\hline & p.N390S & & \\
\hline & c. $444 \mathrm{G}>\mathrm{A} / \mathrm{c} .706 \mathrm{C}>\mathrm{T}(1$ case $)$ & No (this study) & \\
\hline & p.W148*/p.R236* & & \\
\hline \multicolumn{4}{|l|}{ Defects in AchR } \\
\hline \multirow[t]{5}{*}{ CHRNE } & $\begin{array}{l}\text { ع1206ins19 (2 cases) } \\
\text { (c.1248_1266dup19) \#\# }\end{array}$ & Yes & Ohno K. Ann Neurol. 1998; 44:234-241. \\
\hline & $\varepsilon 70$ insG ( 2 cases $)$ & Yes & Ohno K. Ann Neurol. 1998; 44:234-241. \\
\hline & $(\text { c.130dupG) })^{\# \#}$ & & \\
\hline & $\varepsilon 70$ insG/عIVS7+2T>C ( 1 case $)$ & Yes & Ohno K. Ann Neurol. 1998; 44:234-241. \\
\hline & $(\text { c. } 130 \mathrm{dupG} / \mathrm{c} .802+2 \mathrm{~T}>\mathrm{C})^{\# \#}$ & & \\
\hline
\end{tabular}




\begin{tabular}{|c|c|c|c|}
\hline Classification of CMS & Mutations & Published cases & References \\
\hline CHRND & 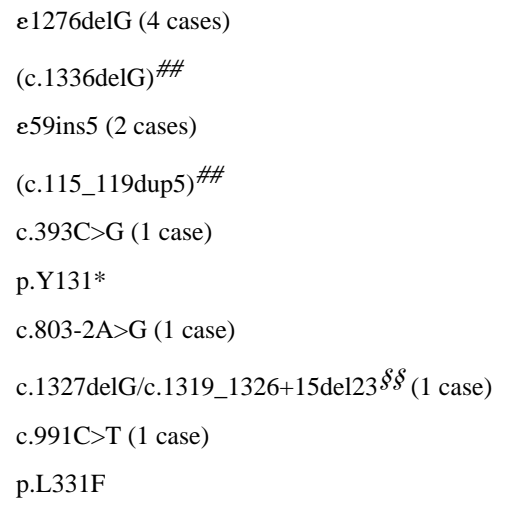 & $\begin{array}{l}\text { Yes } \\
\text { Yes } \\
\text { No (this study) } \\
\text { No (this study) } \\
\text { No (this study) } \\
\text { No (this study) }\end{array}$ & $\begin{array}{l}\text { Ohno K. Ann Neurol. 1998; 44:234-241. } \\
\text { Ohno K. Ann Neurol. 1998; 44:234-241. }\end{array}$ \\
\hline \multicolumn{4}{|l|}{$\begin{array}{l}\text { Defects in endplate } \\
\text { development and } \\
\text { maintenance }\end{array}$} \\
\hline$A G R N$ & $\begin{array}{l}\text { c. } 5023 \mathrm{G}>\mathrm{A}(1 \text { case }) \\
\text { p.G1675S }\end{array}$ & Yes & $\begin{array}{l}\text { Karakaya M, et al. Neuromuscul Disord. } \\
\text { 2014; 24:843. }\end{array}$ \\
\hline MUSK & $\begin{array}{l}\text { c.114T >A ( } 2 \text { cases }) \\
\text { p.D38E }\end{array}$ & Yes & $\begin{array}{l}\text { Gallenmüller C, et al. Neuromuscul } \\
\text { Disord. 2014; 24:31-35. }\end{array}$ \\
\hline \multicolumn{4}{|l|}{$\begin{array}{l}\text { The origin of } \\
\text { neurotransmission defect } \\
\text { is unknown }\end{array}$} \\
\hline GFTP1 & c. $686-2 \mathrm{~A}>\mathrm{G}(1$ case $)$ & No (this study) & \\
\hline
\end{tabular}

\#\# : annotation according to the HGVS guidelines

${ }^{\xi}:$ : c.1319_1326+15delCCGGCGAGGTGGGACAGGAGCCA 